\title{
Estimation of prevalence and incidence of infantile spasms in Taiwan using capture-recapture method
}

\author{
Chih-Chuan Chen ${ }^{\text {a }}$, Ta-Fu Chen ${ }^{\text {a }}$, Haung-Chi Lin ${ }^{\text {b,c }}$, Pei-Ching Oon ${ }^{\text {d,e }}$, \\ Hui-Min $\mathrm{Wu}^{\mathrm{e}}$, Pen-Jung Wang ${ }^{\mathrm{b}, \mathrm{f}}$, Tony Hsiu-Hsi Chen ${ }^{\mathrm{e}, 1}$, Horng-Huei Liou ${ }^{\mathrm{a}, *}$ \\ a Department of Neurology and Pharmacology, National Taiwan University Hospital and National Taiwan University College of Medicine, \\ No. 1, Sec 1, Jan-Ai Road, Taipei 100, Taiwan, ROC \\ ${ }^{\mathrm{b}}$ Department of Pediatrics, National Taiwan University Hospital and National Taiwan University College of Medicine, Taipei, Taiwan, ROC \\ ${ }^{\mathrm{c}}$ Department of Pediatrics, En Chu Kong Hospital, No. 399 Fuhfing Road, Sanhsia, Taipei 237, Taiwan, ROC \\ ${ }^{\mathrm{d}}$ Department of Internal Medicine, En Chu Kong Hospital, Taipei, Taiwan, ROC \\ ${ }^{\mathrm{e}}$ Graduate Institute of Preventative Medicine, College of Public Health, National Taiwan University, \\ No. 19, Hsu-Chow Road, Taipei 100, Taiwan, ROC \\ ${ }^{\mathrm{f}}$ Department of Pediatrics, College of Medicine, Tzu Chi University, No. 701, Section 3, Chung-Yan Road, Hualien 970, Taiwan, ROC
}

Received 5 August 2003; received in revised form 2 December 2003; accepted 15 December 2003

\begin{abstract}
Purpose: To estimate the prevalence, incidence, and case-fatality of infantile spasms (IS) in Taiwan.

Methods: A retrospective cohort of patients with IS was obtained from one medical center to identify 69 IS cases from 1985 to 1997. This cohort, in conjunction with the claimed data from the National Health Insurance, was used to estimate the prevalence and incidence of IS by capture-recapture design, taking the case-fatality of IS into account.

Results: The prevalence rate of IS for aged 0-9 years was 0.046 per thousand. The incidence rate was estimated as 6 over 100,000 per year in Taiwan. Of the 69 IS cases, 8 deaths were ascertained. The case-fatality rate was $11.6 \%$. The leading cause of death was status epilepticus.

Conclusions: We have demonstrated an efficient method to estimate the incidence and prevalence rates of IS in Taiwan. Our results help to make a clear understanding of the disease burden of IS in this society.
\end{abstract}

(C) 2004 Elsevier B.V. All rights reserved.

Keywords: Infantile spasms; Prevalence; Incidence; Capture-recapture; Epidemiology

\footnotetext{
* Corresponding author. Tel.: +886-2-23123456x8325; fax: +886-2-23915297.

E-mail addresses: stony@episerv.cph.ntu.edu.tw (T.H.-H. Chen), hhliou@ha.mc.ntu.edu.tw (H.-H. Liou).

${ }^{1}$ Co-corresponding author. Tel.: +886-2-23587620; fax: $+886-2-23587707$.
}

\section{Introduction}

Although infantile spasms (IS) accounts for only about $2 \%$ of all cases of childhood epilepsy (Hauser, 1994), it is one important type of epilepsy with unique clinical presentations. Previous studies showed factors affecting the risk of IS were highly related to the underlying neurological abnormalities or pre- and perinatal insults (Rantala et al., 1996; Liou et al., 2001). 
The prevalence rate of IS has been reported to range from 0.14 to 0.2 per thousand children aged from birth through 9 years (Cowan and Hudson, 1991; Trevathan et al., 1999). Males had 1.5-fold risk for IS as compared with females (Cowan et al., 1989). Annual incidence rate of IS was reported to range from 16 to 42 over 100,000 live births per year (Riikonen and Donner, 1979; Cowan and Hudson, 1991; Ludvigsson et al., 1994; Trevathan et al., 1999).

Most studies on IS have been conducted in western countries but few in oriental countries. In addition, few descriptive studies were designed to simultaneously estimate the incidence and the prevalence of IS, taking mortality into account. However, conducting a population-based survey to estimate descriptive epidemiological profiles for a rare disease is costly and time-consuming. To tackle this problem, we proposed an efficient capture-recapture method (Bobo et al., 1994; Murphy et al., 1995; O'Callaghan et al., 1998; Debrock et al., 2000) in conjunction with epidemiological study design to estimate the prevalence and incidence rate of IS, taking cases of death into account.

Two data resources were available for such a purpose. One was based on primary data using a retrospective cohort from a medical center, National Taiwan University Hospital (NTUH). To take cases of death into count, this cohort can be linked to the mortality registry to estimate the survival of patients with IS after its onset. The other relies on the clinical data from the National Health Insurance (NHI) system that has been implemented in Taiwan since 1995. Although NHI system covers $95 \%$ population and can be used to estimate the prevalence of IS, whether case-ascertainment, particular in early stage, was complete is worthy of being investigated. The aim of our study was therefore to use a retrospective cohort of IS from one medical center in Taiwan to estimate the incidence, prevalence and case-fatality of IS through capture-recapture design whereby information on this cohort was compared with the data from NHI that may have incomplete ascertainment of IS.

\section{Methods}

\subsection{Study design}

NTUH is a 2000-bed teaching hospital and one of the major tertiary referral centers in Taiwan. There

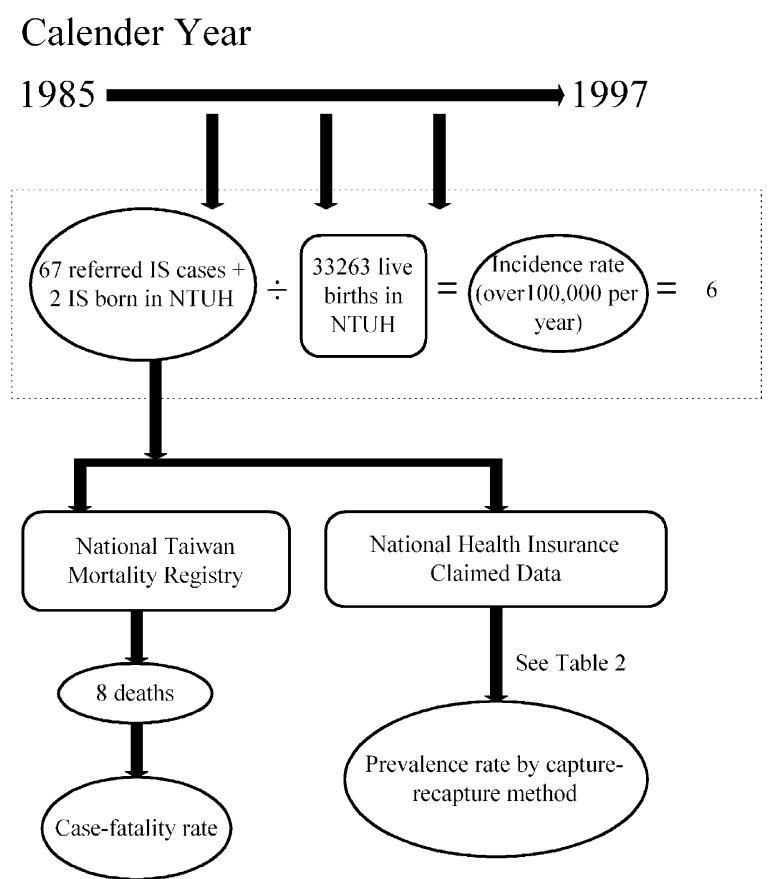

Fig. 1. Study design for estimating prevalence, incidence and case-fatality rates.

are nine medical centers on the Taiwan Island. As all could diagnose and treat of IS, not all IS were remitted to NTUH. We therefore used a capture-recapture method in conjunction with a retrospective cohort. Fig. 1 shows the study design for estimating the prevalence rate, incidence rate and case-fatality of IS. We identified IS from NTUH during the period from 1985 to 1997. Primary data from NTUH were compared with NHI claimed data, making allowance for cases of death by linking NTUH cohort data with Taiwan Mortality Registry. Details of the study design are depicted in the following:

(1) Prevalence rate: There were two datasets used for estimating the underlying prevalence rate of IS. The major dataset was derived from a retrospective cohort consisting of a total of 69 IS patients that had been diagnosed as IS by pediatric neurologists from 1985 to 1997 in NTUH, the teaching hospital that captured a substantial proportion of IS cases in Taiwan. In order to estimate nation-wide prevalence rate of IS in Taiwan, a nation-wide data from the NHI was also accrued, 
including 35 IS and 3,064,804 children aged 0-9 years derived from claimed data on NHI in 1997. However, as data were available from only 1 year and some IS cases may be under-ascertained in early period of NHI, a capture-recapture method (see the description later) was used to estimate the nation-wide prevalence rate by combining the two datasets.

(2) Case-fatality rate: Linking the above retrospective cohort with the national Taiwan mortality registry gives the details of information on death among the 69 IS cases. This provided the base for calculating the case-fatality rate of IS.

(3) Incidence rate per 100,000 live birth: A retrospective cohort was also used to estimate the annual incidence rate for the underlying population who will seek for maternity care in NTUH by calculating numbers of IS cases delivered at NTUH divided by a total of live births at NTUH during the same period.

\subsection{Diagnosis of IS}

The diagnosis of IS was made by the presence of a clinical history of spasms, and a characteristic electroencephalogram (EEG) with or without psychomotor retardation after the onset of spasms (Lacy and Penry, 1976; Hrachovy and Frost, 1989). The spasms were classified as flexor, extensor or mixed pattern according to Kellaway et al. (1979). The EEG was classified as either hypsarrhythmic or modified hypsarrhythmic by the criteria of Gibbs and Gibbs (1952) and Hrachovy et al. (1984), respectively. In addition, subjects having spasms and psychomotor retardation with multifocal EEG activity were included. According to the 1989 Classification of Epilepsy and Epileptic Syndromes of the International League Against Epilepsy (Commission, 1989), patients were decided into symptomatic and cryptogenic groups. For etiological investigation, every patient received thorough clinical history evaluation, developmental and neurological assessment, neuroimaging such as computed tomography or magnetic resonance imaging of the head, chromosomal studies and metabolic studies, such as serum amino acids and urine organic acids. Cryptogenic group was characterized by (1) normal pregnancy and birth, (2) normal development before the onset of spasms and absence of neurological ab- normalities at the onset of spasms, (3) absence of any other type of seizure before the onset of spasm, and (4) normal laboratory and neuroimaging findings at onset (Watanabe, 1998). When an etiology or signs of previous brain damage could be found or the above criteria were not fulfilled, the patient was classified as symptomatic group.

\subsection{Statistical methods}

The principle of capture-recapture is that the size of a population can be estimated by trapping and marking a sample and then releasing the marked sample and allowing it to mix with the other members of the population before trapping a second sample. The ratio of marked samples to all individuals in the second catch is the same as the ratio of the number in the first catch to the number in the whole population. To estimate the nation-wide prevalence, a simple capture-recapture design was used to combine the retrospective cohort with the claimed data on NHI. The procedure is described as follows: Suppose 69 subjects who were diagnosed as IS during the period from 1985 to 1997 were captured by NTUH, labeled with a mark "NTUH-IS" and released into the population pool. The NHI nationwide data recaptured these cases. Since not all 69 subjects were recaptured by NHI, the ratio of recaptured cases with the mark "NTUH-IS" to all cases identified from NHI in conjunction with 69 subjects was used to indirectly estimate nationwide IS cases. The detailed calculations are shown in Table 2. Let $N_{11}, N_{10}, N_{01}, N_{00}$ be denoted as IS cases present in both data resources $\left(N_{11}\right)$, NTUH but not NHI $\left(N_{10}\right)$, NHI but not NTUH $\left(N_{01}\right)$ and absent from both data sets $\left(N_{00}\right)$. The purpose of capture-recapture is to first estimate $N$ which can be estimated by $N_{._{1}} \times N_{1 .} / N_{11}$. The number of under-ascertainment, $N_{00}$, was therefore estimated by subtracting $N_{11}, N_{10}$, and $N_{01}$ from $N$.

\section{Results}

Table 1 shows the distribution of age, sex and etiologies in IS cases. Male and female were equally affected. The peak age of IS onset was between 4 and 9 months after birth. Among the 69 cases, 55 cases (79.7\%) were symptomatic and 14 cases $(20.3 \%)$ were 
Table 1

Distribution of age, gender and etiology of 69 infantile spasms (IS) cases captured by NTUH

\begin{tabular}{lcc}
\hline & \multicolumn{2}{l}{ Case number (\%) } \\
\cline { 2 - 3 } & Male & Female \\
\hline Age (years) & 5 & 4 \\
$0-3$ & 10 & 15 \\
$4-6$ & 11 & 10 \\
$7-9$ & 8 & 6 \\
$\geq 10$ & $34(49 \%)$ & $35(51 \%)$ \\
Total & $55(79.7 \%)$ & \\
Symptomatic & 9 & \\
Tuberous sclerosis & 8 & \\
Asphyxia & 7 & \\
CNS malformation & 6 & \\
Prematurity & 5 & \\
Neurodegenerative & 4 & \\
CNS infection & 4 & \\
Traumatic brain injury & 3 & \\
Chromosome abnormality & 3 \\
Early infantile epileptic & \\
$\quad$ encephalopathy & 1 & \\
Pyridoxine dependent seizure & 1 & \\
Hypoxic-ischemic & \\
encephalopathy, postnatal & & \\
Hypomelanosis & 1 & \\
Unidentified with cerebral palsy & 3 \\
Cryptogenic & $14(20.3 \%)$ & \\
\hline
\end{tabular}

cryptogenic. The associated diseases of the 55 symptomatic cases were also listed in the Table 1.

Linking the NTUH cohort with the NHI data yields the result of the estimated prevalence rate of IS by the capture-recapture method (Table 2). Seventeen cases $\left(N_{11}\right)$ were both captured by NHI data and NTUH cohort. Eighteen cases $\left(N_{01}\right)$ were found in NHI but not in NTUH. Fifty-two cases $\left(N_{10}\right)$ were identified from NTUH but not captured by NHI data. Cases that were not captured in both datasets $\left(N_{00}\right)$ were estimated as 55 .

The application of capture-recapture method yields $142(35 \times 69 / 17)$ estimated prevalent cases of IS $(N)$ from 1985 to 1997 in Taiwan. The number of the denominator was $3,064,804$. Hence, the prevalence rate of IS aged 0-9 years could be estimated as 0.046 per thousand. It should be noted that of 142 IS cases estimated from capture-recapture method, both found in two data source accounted for $11.97 \%$ (17). Cases found in NHI but not in NTUH explained 12.68\% (18). Cases found in NTUH but not in NHI were $36.61 \%$ (52). Those cases not found in both data sources were $38.73 \%$ (55)

There were only 2 out of 69 IS cases were delivered in NTUH, and the rest of the cases were referred from other hospitals. There were a total of 33,263 live births in NTUH in the study period. The incidence rate of IS was estimated as 6 over 100,000 live births per year.

Of the 69 IS cases, there were eight deaths (five males and three females) ascertained after the linkage with the Taiwan Mortality Registry. The case-fatality rate was $11.6 \%$. The estimated death rate per year using the exponential distribution was 0.01 per case. Cause of death in these patients was status epilepticus (4), pneumonia (1), accident (1) and unknown (2).

\section{Discussion}

\subsection{Credibility of results}

Our study used capture-recapture method in combination with a retrospective cohort design and NHI data to estimate disease prevalence and incidence in Taiwan, taking case-fatality rate of IS into account. The results showed that the estimated prevalence rate for

Table 2

Estimates of prevalence of infantile spasms (IS) in Taiwan with capture-recapture method

\begin{tabular}{llll}
\hline & Cases present in & Cases absent in & Total \\
& NHI database & NHI database & $69\left(N_{1 .}\right)$ \\
\hline Cases present in NTUH-IS cohort & $17\left(N_{11}\right)$ & $52\left(N_{10}\right)$ & $73\left(\right.$ estimated $\left.N_{0}\right)$ \\
Cases absent in NTUH-IS cohort & $18\left(N_{01}\right)$ & 55 (estimated $\left.N_{00}\right)$ & $142($ estimated $N)$ \\
Total & $35(N \cdot 1)$ & 107 (estimated $\left.N_{.0}\right)$ & . \\
\hline
\end{tabular}

(1) Assumption: consultation habits of all IS patients do not change from year to year. Therefore, $N=35 \times 69 / 17=142$, and $N_{00}=55$. (2) A total of 3,064,804 children aged between 0 and 9 years old were presented in NHI data in 1997. Hence, prevalence of IS = $142 / 3,064,804=0.046 / 1000$. 
children aged less than 10 was 0.046 per thousand and annual incidence rate was 6 over 100,000 live birth per year. The case-fatality rate was estimated as $11.6 \%$.

To examine the validity of our results, we applied annual incidence rate per live birth and case fatality rate to estimate nation-wide prevalence rate. We compared this estimate with that calculated from capture-recapture method. As we had around 280,000 live births per year for the birth cohort from 1985 to 1997 among children aged 0-9 years, this yields 168 IS on the basis of 6 over 100,000 liver births per year. As the annual death rate was 0.01 per case, this gives 17 deaths among the 168 IS cases. After subtracting these cases, the estimated prevalent cases for 0-9 years old were 151 that are close to our estimate from capture-recapture method. This suggests that our estimated prevalence, incidence and case fatality may be representative of current epidemiological profile of IS in Taiwan.

It could be argued that the characteristics of age groups from NTUH may be different from those used in this validation. Subjects from NTUH may be more likely to yield more IS cases than the latter. The slightly higher estimated cases in validated sample in comparison with the estimate from capture-recapture method can support this argument. However, as the difference is trivial, this problem is not serious.

\subsection{Methodological consideration}

From methodological viewpoint, our study design was efficient in estimating the magnitude of morbidity and mortality of IS. A retrospective cohort plus the NHI data using the capture-recapture method provides an efficient way to estimate the prevalence and incidence of IS. This method dispenses with using a large nationwide survey for estimating these basic epidemiological figures.

Another strength of using capture-recapture method in our study is to detect the degree of underascertainment of NHI system, particularly in early period. In our study, there were $36.6 \%$ (52) cases ascertained in NTUH but not found in NHI system. This suggests that the direct use of NHI system for estimation of prevalence of IS may lead to an underestimate.

It could be also argued that the estimation using the capture-recapture method requires some assumptions. For instance, it was assumed that the probabil- ity of being listed in NHI system was independent of that identified in NTUH. In addition, we also assumed that the characteristics of IS cases in other hospitals in the same period and not listed in NHI system would be similar to that of the 52 IS cases in NTUH and not identified in NHI system. These two assumptions were not unreasonable since the NHI system covered almost $95 \%$ of the population in Taiwan and subjects with IS seeking medical services might be rather similar.

Although capture-recapture method provides an efficient method for the estimation of prevalence as shown in previous studies on epilepsy to cope with incomplete case-ascertainment data (Bobo et al., 1994; Hook and Regal, 1995; Murphy et al., 1995; Debrock et al., 2000). It is still possible subject to the underestimation of IS due to cases of death, particularly in a retrospective analysis. To cope with this problem, we used a retrospective cohort study design to take this concern into account. Results of case-fatality rate give $12 \%$ attrition due to death during 13-year follow-up. The underestimation could be possible without using this design. However, the estimates of incidence from NTUH data may also be skewed if the obstetric service caters to high risk pregnancies. This may lead to an underestimates of IS. Nevertheless, we believe the impact of mortality on the underestimates of IS is minor because only $12 \%$ attrition due to death was ascertained.

\subsection{Comparison with other studies}

The prevalence and incidence rates of IS estimated in our study were far lower than those from Western countries, such as in Finland (Riikonen and Donner, 1979), Atlanta, GA, USA (Trevathan et al., 1999) and Tokyo, Japan (Tsuboi, 1988). We believe lower incidence or prevalence of IS compared with other countries is not due to methodological problem but possible due to two causes. First, there are certainly other epilepsy centers in Taiwan and the assumption that NTUH has the potential to see all IS cases may be an overestimate. This could explain the lower estimates in our results compares with those from the previous studies. Second, underdiagnosis is the other possibility. It should be noted capture-recapture method can cope with incomplete ascertainment of IS cases but can not solve the problem of under- 
diagnosis. The problem of underdiagnosis should be explored in the future study.

The case-fatality rate reported from previous studies (Lacy and Penry, 1976; Hrachovy and Frost, 1989; Matsumoto et al., 1981; Ohtahara, 1984) was between 5 and $22 \%$, and that in our study was approximately $12 \%$. This finding suggested that the quality of treatment on IS in Taiwan may not be worse than that in western countries. Among deaths of IS cases, status epilepticus is the leading cause of death, which underscores the importance of advanced seizure control.

In conclusion, we have demonstrated an efficient method to estimate the prevalence and incidence rate of IS in Taiwan. These estimates enable one to make a clear understanding of the impact of the disease burden of IS in this society. Using capture-recapture method is helpful for the association of ascertainment of IS and medical care system, such as NHI.

\section{Acknowledgements}

This work was supported by grants NSC 89-2314B-002-145 from the National Science Council and NTUH 91-S-072, 91-A-21-3 from National Taiwan University Hospital.

\section{References}

Bobo, J.K., Thapa, P.B., Anderson, J.R., Gale, J.L., 1994. Acute encephalopathy and seizure rates in children under age two years in Oregon and Washington State. Am. J. Epidemiol. 149, 27-38.

Commission on Classification and Terminology of the International League Against Epilepsy, 1989. Proposal for revised classification of epilepsies and epileptic syndromes. Epilepsia 30, 389-399.

Cowan, L.D., Hudson, L.S., 1991. The epidemiology and natural history of infantile spasms. J. Child Neurol. 6, 355-364.

Cowan, L.D., Bodensteiner, J.B., Leviton, A., Doherty, L., 1989. Prevalence of the epilepsies in children and adolescents. Epilepsia 30, 94-106.

Debrock, C., Preux, P.M., Houinato, D., Druet-Cabanac, M., Kassa, F., Adjien, C., Avode, G., Denis, F., Boutros-Toni, F., Dumas, M., 2000. Estimation of the prevalence of epilepsy in the Benin region of Zinvie using the capture-recapture method. Int. J. Epidemiol. 29, 330-335.
Gibbs, F.A., Gibbs, E.L., 1952. Atlas of Electroencephalography. II. Epilepsy. Addision-Wesley, Reading, MA.

Hauser, W.A., 1994. The prevalence and incidence of convulsion disorders in children. Epilepsia 35 (Suppl. 2), S1-S6.

Hook, E.B., Regal, R.R., 1995. Capture-recapture methods in epidemiology methods and limitations. Epidemiol. Rev. 17, 243-264

Hrachovy, R.A., Frost Jr., J.D., 1989. Infantile spasms: a disorder of the developing nervous system. In: Kellaway, P., Noebels, J.L. (Eds.), Problems and Concepts in Developmental Neurophysiology. Johns Hopkins University Press, Baltimore, MD, pp. 131-147.

Hrachovy, R.A., Frost, J.D., Kellaway, P., 1984. Hypsarrhythmia: variations on the theme. Epilepsia 25, 317-325.

Kellaway, P., Hrachovy, R.A., Frost, J.D., Zion, T., 1979. Precise characterization and quantification of infantile spasms. Ann. Neurol. 6, 214-218.

Lacy, J.R., Penry, J.K., 1976. Infantile Spasms. Raven Press, New York.

Liou, H.H., Oon, P.C., Lin, H.C., Wang, P.J., Chen, T.H.H., 2001. Risk factors associated with infantile spasms: a hospital-based case-control study in Taiwan. Epilepsy Res. 47, 91-98.

Ludvigsson, P., Olafsson, E., Sigurthardottir, S., Hauser, W.A., 1994. Epidemiologic features of infantile spasms in Iceland. Epilepsia 35, 802-805.

Matsumoto, A., Watanabe, F., Negoro, T., Sugiura, M., Iwase, K., Hara, K., Miyazaki, S., 1981. Long-term prognosis after infantile spasms: a statistical study of prognosis factors in 200 cases. Dev. Med. Child. Neurol. 23, 51-65.

Murphy, C.C., Trevathan, E., Yeargin-Allsopp, M., 1995 Prevalence of epilepsy and epileptic seizures in 10-year-old children: results from the Metropolitan Atlanta Developmental Disabilities Study. Epilepsia 36, 866-872.

O’Callaghan, F.J.K., Shiell, A.W., Osborne, J.P., Martyn, C.N., 1998. Prevalence of tuberous sclerosis estimated by capture-recapture analysis. Lancet 351 (9114), 1490.

Ohtahara, S., 1984. Seizure disorders in infancy and childhood Brain Dev. 6, 509-519.

Rantala, H., Shields, W.D., Christenson, P.D., Nielsen, C., Buch, D., Jacobsen, V., Zachau-Christiansen, B., Uhari, M., Cherry, J.D., 1996. Risk factors of infantile spasms compared with other seizures in children under 2 years of age. Epilepsia 37, 362-366.

Riikonen, R., Donner, M., 1979. Incidence and etiology of infantile spasms from 1960 to 1976: a population study in Finland. Dev. Med. Child. Neurol. 21, 333-343.

Trevathan, E., Murphy, C.C., Allsopp, M.Y., 1999. The descriptive epidemiology of infantile spasms among Atlanta children. Epilepsia 40, 748-751.

Tsuboi, T., 1988. Prevalence and incidence of epilepsy in Tokyo. Epilepsia 29, 103-110.

Watanabe, K., 1998. West Syndrome: etiological and prognostic aspects. Brain Dev. 20, 1-8. 